\title{
Dynamic Background Correction of Electron Backscatter Diffraction Patterns
}

\author{
Damian J. Dingley*, Stuart I. Wright*, Matthew M. Nowell* \\ * EDAX-TSL, 392 E 12300 S, Draper, UT 84020
}

The quality of electron backscatter diffraction (EBSD) patterns can be significantly improved using "flat fielding". Flat fielding is a standard image processing procedure for removing long-range intensity gradients. Flat fielding is performed by first obtaining a background image. For ESBD, this is generally done by scanning the beam over the surface of sample at low magnifications (defined relative to the grain size of the examined material), essentially obtaining an average pattern without any crystallographic detail by sampling numerous grains of different orientations. A pattern is then obtained by positioning a stationary beam within a single grain. The background pattern is subtracted or divided from the stationary beam pattern. [1-2] This procedure works very well on most polished polycrystalline samples. Flat fielding is particularly helpful when performing automated EBSD scans where an operator cannot optimize individual patterns by manually adjusting the brightness and contrast levels.

There are cases where it can be difficult to obtain an effective background pattern. For instance, in the case of a single crystal where the pattern averaged over a large scan area is nearly identical to the pattern with the beam stationary. In this case, a background pattern can sometimes be obtained by defocusing the electron beam or rotating the sample while the beam is collecting the background reference. Another case is on samples that cannot be polished and have rough surfaces such as facture surfaces or as-deposited thin films. Because of this topography, the intensity gradient within the image may change dramatically from point-to-point. Multiphase materials can also be difficult when the overall intensity can significantly change from one phase to another due to changes in the average atomic weight. In these cases, it is often more effective to use a technique termed "soft" flat fielding or dynamic background correction. In this case, the background pattern used in the flat fielding procedure is formed from the pattern itself. This is done by blurring the pattern using a relatively large radius Gaussian convolution mask. For an example see Tao [3]. This technique is considerably more effective when performed at higher pixel bit depths, i.e. 12 bit vs. conventional 8 bit.

While this technique can generally improve pattern quality over a larger fraction of the sampled area, particularly from rough surfaces, it does present a few drawbacks over the conventional approach. Any defects in the phosphor screen such as scratches or pores are not removed using the dynamic approach whereas the conventional approach is very effective at removing them. Another drawback is the image processing time required to create the blurred background which results in longer pattern collection times. This is particularly relevant during orientation imaging, where hundreds of thousands of EBSD patterns are automatically collected and processed. A less obvious drawback of this technique is the impact on image quality maps. As each pattern in a scan is dynamically flatfielded the average pattern intensity is essentially normalized. This leads to a loss in contrast in the image quality maps. However, other image quality measures [3] could be employed to overcome this deficiency.

[1] S. I. Wright and B. L. Adams, Met. Trans. A., 23 (1992) 759.

[2] J. R. Michael and R. P. Goehner, MSA Bulletin 23 (1993) 168.

[3] X. Tao, Ph. D. Thesis, Lehigh University (2004). 

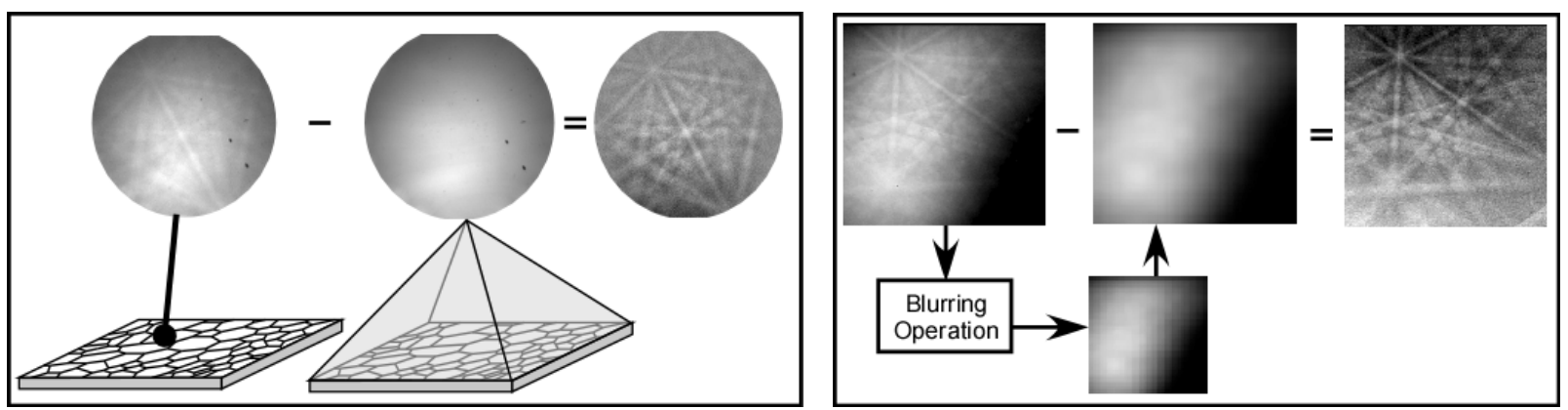

FIG. 1. Conventional (left) and dynamic (right) background correction flow charts.
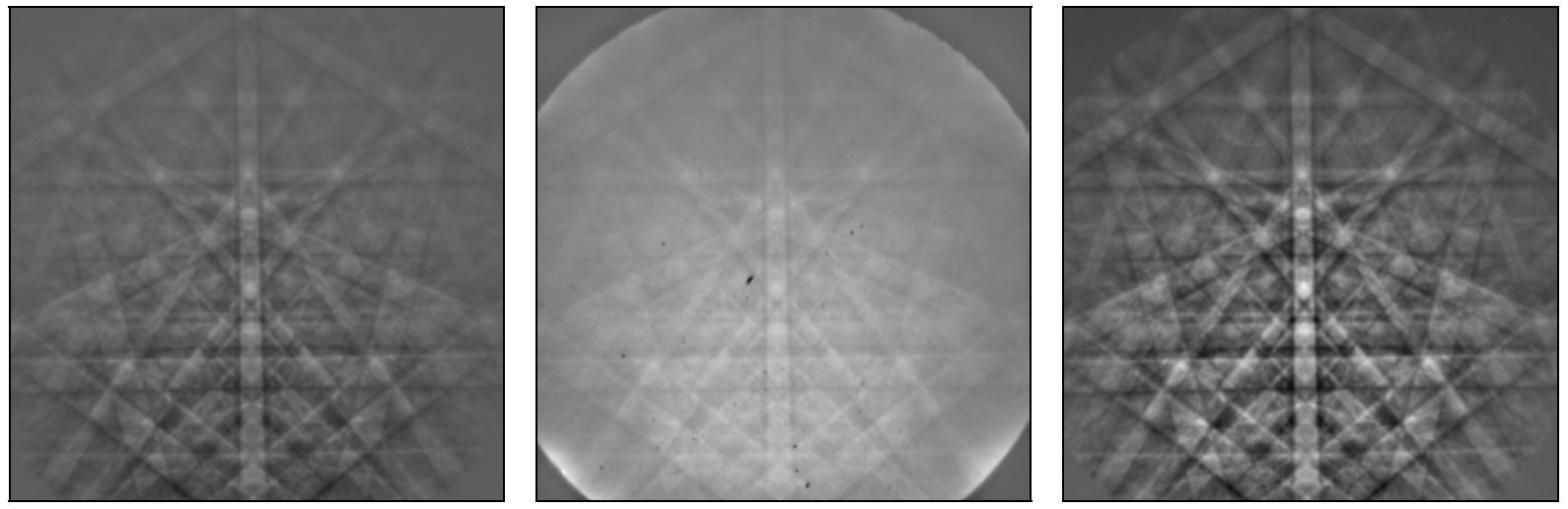

FIG. 2. EBSD patterns collected from a single crystal 4H SiC sample using conventional (left), dynamic (center) and combined (right) background correction.
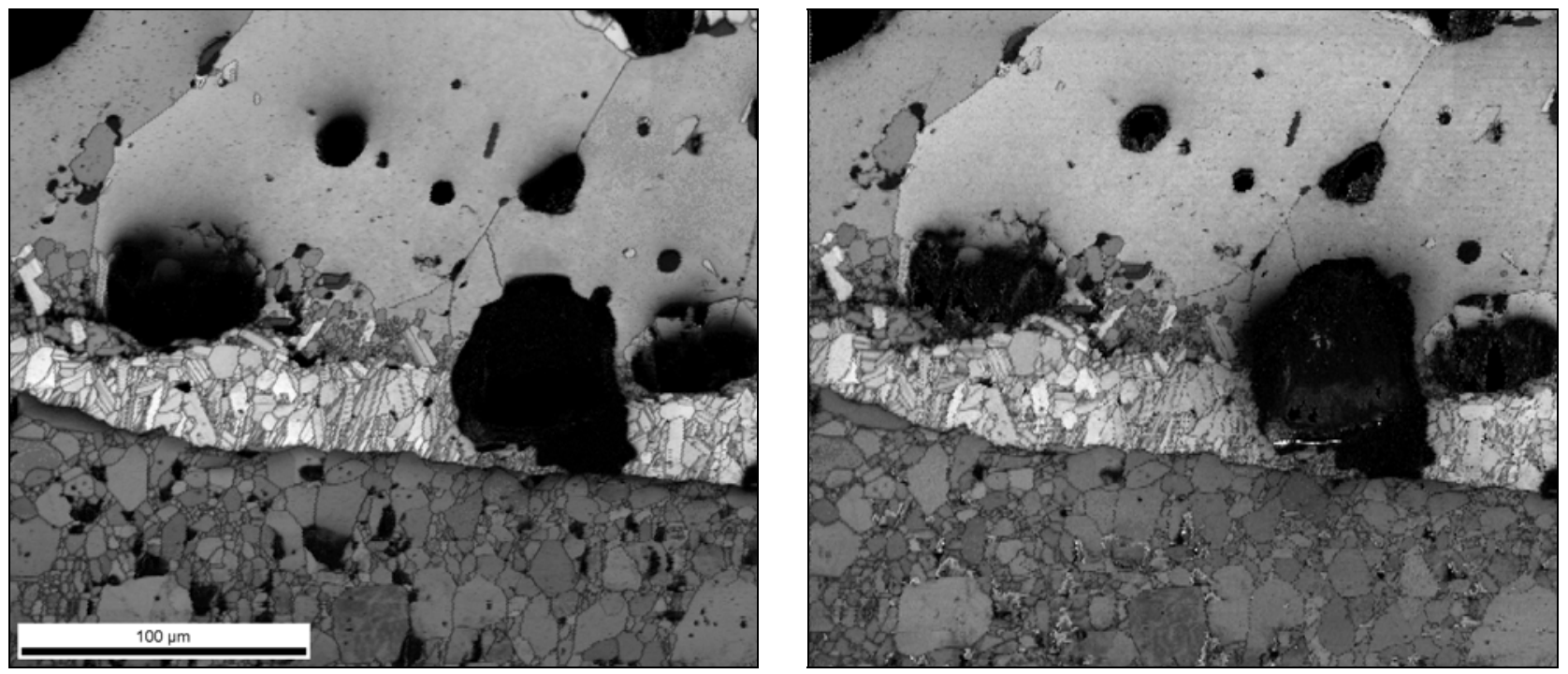

FIG. 3. Image quality maps generated from a multiphase ceramic sample containing $\mathrm{CuO}, \mathrm{CuAlO}_{2}$, $\mathrm{CuAl}_{2} \mathrm{O}_{4}$, and $\mathrm{Al}_{2} \mathrm{O}_{3}$ using conventional flat-fielding (left) and with dynamic (right) background correction. 